\title{
Curcumin nanoformulations as potential antimicrobial agent
}

\begin{abstract}
Turmeric has been widely used as antimicrobial agent in Asian countries for centuries. The demand for a safe and reliable antimicrobial agent has increased world over due to the side effects of existing drugs and the development of newer drug-resistant microbes. Curcumin, the active ingredient of turmeric, has come up as a potential solution to these problems. This research article summarizes the recent developments in the field of curcumin nanoformulations and their applications as antimicrobial agent
\end{abstract}

Keywords: curcumin, nanoformulations, antimicrobial activity, turmeric
Volume 5 Issue 5 - 2017

\author{
Sonia Tyagi, Javed A Farooqi \\ School of Sciences, Indira Gandhi National Open University, \\ India
}

Correspondence: Javed A Farooqi, Professor, School of Sciences, Indira Gandhi National Open University, Maidan Garhi, New Delhi- I 10068, India, Tel 01 I 29572822,

Email jafarooqi@ignou.ac.in

Received: August 31, 2017| Published: November 13, 2017
Abbreviations: E. Coli, escherichia coli; LED, light emitting diode; B. macerans, bacillus macerans; B. licheniformis, bacillus licheniformis; PVA, polyvinyl acetate

\section{Introduction}

Globally, a very large human population has been affected by microbes like bacteria, fungi, yeast, protozoa etc. This has led to prolonged and costly treatment and mortality risk. Scientists have come up with numerous advancements in drug-development newer anti-microbial agents to combat these infections but over a period of time, the drug-resistant organisms evolve making the drug ineffective. Hence, a need of effective anti-microbial agent has been felt for long.

The public interest in traditional medicine, particularly plant based medicine, has grown to a great extent due to their known and proven medicinal properties and limited or no side effects. This has led to an extensive research on the potential of using natural origin substances in human healthcare. Hundreds of studies were conducted to investigate the effect of herbs and spices on human health and their impact on prevention and treatment of chronic diseases. Turmeric (Curcuma longa) is a perennial Indian herb with large aromatic rhizomes that has been used for centuries in India and China as traditional herbal remedy ${ }^{1}$ and victory spice. Turmeric has been used traditionally for treatment of lives diseases, bacterial infections, skin disorders, cough, asthma, osteoarthritis, heart diseases and indigestion..$^{2-6}$ It has been used in medicinal form in two ways: as topical application for the treatment of wounds, tumours and inflammation and by oral intake for the treatment of wide variety of diseases such as asthma, anorexia, sour throat, cough and cold. ${ }^{7,8}$ Turmeric has been used as a home remedy against microbial infections in many Asian countries. Its antimicrobial activity has been widely reported in literature. ${ }^{9}$ Curcumin (diferuloylmethane with chemical name of (1,7-bis(4hydroxy-3-methoxyphenyl)-1,6-heptadiene-3,5-dione)) is the main ingredient of turmeric. It is highly potent, nontoxic and bioactive. Curcumin has been found to be an effective antibacterial agent against bacteria such as Bacillus subtilis, B. macerans, B. licheniformis, and Azotobacter ${ }^{10}$ and Staphylococcus aureus, Salmonella paratyphi. ${ }^{11}$ The effectiveness of Curcumin against 20 types of Candida species ${ }^{12}$ has also been reported. The main reason for the limited application of curcumin as antimicrobial agent has been its poor aqueous solubility which adversely affects its bioavailability. In the past two decades, the scientists have found its solution in nanotechnology. A variety of nanoparticles formulations have been developed which have demonstrated the enhanced effectiveness of curcumin as an antimicrobial agent.

\section{Antimicrobial activity}

Numerous studies have reported the effectiveness of curcumin nanoformulations against different microbes at a very low concentration. Gopal $\mathrm{J}$ et al., ${ }^{13}$ compared the bactericidal property of macro, micro and nanocurcumin against four bacteria: E. Coli, Salmonella enteritidis 12021, Streptococcus mutans 11823 and Staphylococcus aureus sub. aureus and demonstrated the enhanced bactericidal activity of nanocurcumin when compared to macro and micro curcumin. The nanoformulations of curcumin was prepared using wet milling technique. Vimala et al., ${ }^{14}$ have fabricated Curcumin encapsulated chitosan-PVA silver nanocomposite films by in situ fabrication of chitosan-PVA-silver nanocomposite films and demonstrated its effectiveness against E. Coli, Pseudomonas, Staphylococcus, Micrococcus, Candida albicans, and P. aeruginosa by disk diffusion method. The antimicrobial effectiveness of formulated curcumin is confirmed as clear zone of inhibitions are formed in case of formulated curcumin. A very interesting study of LED-activated curcumin-gold nanoparticles by Courrol et al., ${ }^{15}$ tested the effectiveness of curcumin nanoformulations against Gram positive and Gram negative bacteria. This nanoformulations of gold and curcumin was prepared by mixing, stirring and illuminating the mixture of chloroauric acid, PEG and curcumin. The study showed $98 \%$ inhibition at $30 \mu \mathrm{g} / \mathrm{ml}$ nanoformulations concentration for both Gram positive and Gram negative bacteria. The study by Johromi et al., ${ }^{16}$ demonstrated the effectiveness of curcumin-loaded chitosan-TPP nanoparticles, prepared by ionotropic gelation method, in treating the infections caused by Staphylococcus aureus and Pseudomonas aeruginosa.

The enhanced effectiveness of curcumin nanoformulations prepared by wet milling technique was also demonstrated by Bhawna et al. ${ }^{17}$ against Staphylococcus aureus, Bacillus subtilis, Escherichia coli, Pseudomonas aeruginosa, Penicillium notatum and Aspergillus niger. Their study proved the superior solubility of curcumin as nanoformulations and elevated effect against these Gram positive and negative bacterial and fungal stains. The study by Shlar et al., ${ }^{18}$ of 
curcumin nanoparticles against $E$. Coli proves the effectiveness of curcumin nanoparticles as antibacterial agent even at a concentration as low as $500 \mu \mathrm{M}$. The curcumin nanoformulations used in this study was prepared using antisolvent precipitation technique. The study of Adahoun et al., ${ }^{19,20}$ proved better effectiveness of curcumin nanoparticles against Gram positive bacteria as compared to Gram negative bacteria. For this study, they used the nanocurcumin in combination with $8 \mathrm{mT}$ AC static magnetic field. They studied the curcumin nanoformulation effectiveness against two gram-positive (Micrococcus luteus Staphylococcus aureus) and two gram-negative (Escherichia coli, Pseudomonas aeruginosa). The selected grampositive bacteria had higher sensitivity than the selected gramnegative bacteria for both curcumin and nanocurcumin prepared by wet-milling technique.

\section{Conclusion}

All the above studies confirm the enhanced antimicrobial activity of curcumin as nanoformulations. This enhancement in activity of curcumin is due to increased solubility of curcumin resulting in its better bioavailability. Almost all the nanoformulations of curcumin have been found to be effective against Gram positive and Gram negative bacteria's. Also, most of them have shown promising antifungal activity as well, thereby establishing curcumin as a potential antimicrobial agent for further investigations.

\section{Acknowledgements}

We sincerely acknowledge the support provided by Dr. Lalita Kumar, Professor, School of Sciences, Indira Gandhi National Open University (IGNOU), New Delhi, India, in completion of this minireview.

\section{Conflict of interest}

The author declares no conflict of interest.

\section{References}

1. Bhowmik D, Chiranjib K, SampathK, et al. Turmeric: A herbal and traditional medicine. Archives of Applied Science Research. 2009;1(2):86-108.

2. http://botanical.com/site/by_you/article_tumeric/turmeric.html

3. Tyagi P, Singh M, Kumari H, et al. Bactericidal Activity of Curcumin I Is Associated with Damaging of Bacterial Membrane. PLoS One. 2015;10(3):e0121313.

4. http://www.med-health.net/Turmeric-Powder-For-Skin.html
5. http://www.livestrong.com/article/199352-turmeric-for-coughs

6. http://umm.edu/health/medical/altmed/herb/turmeric

7. http://www.turmericforhealth.com/turmeric-benefits/6-benefits-oftopical-turmeric-in-cancer

8. https://breastcanceryogablog.com/2015/11/04/topical-application-ofturmeric-curcumin-for-cancer

9. Moghadamtousi SZ, Kadir HA, Hassandarvish P, et al. A Review on Antibacterial, Antiviral, and Antifungal Activity of Curcumin. BioMed Research International. 2014;2014(2014):12.

10. http://dx.doi.org/10.1155/2014/186864

11. Naz S, Jabeen S, Manzoor S, et al. Antibacterial activity of Curcuma longa varieties against different strains of bacteria. Pak J Bot. 2001;1(42):455-462.

12. Chaudhary N, Sekhhon B. Potential therapeutic effect of curcumin-an update. J Pharma Edu Res. 2012;3(2):64-71.

13. Martins CV, da Silva DL, Neres AT, et al. Curcumin as a promising antifungal of clinical interest. J Antimicro Chemother. 2009;63(2):337339.

14. Gopal J, Muthu M, Chun S. Bactericidal Property of Macro-, Micro- and Nanocurcumin: An Assessment. Arab J Sci Eng. 2016;41(6):2087-2093.

15. Vimala K, Mohan YM, Varaprasad K, et al. Fabrication of Curcumin Encapsulated Chitosan-PVA Silver Nanocomposite Films for Improved Antimicrobial Activity. Journal of Biomaterials and Nanobiotechnology. 2011;2(1):55-64.

16. Courrol DS, Teixeira BH, Pereira CBP, et al. Pegylated Curcumin with Gold Nanoparticles: Antimicrobial Agent Evaluation. Journal of Biomedical Engineering and Biosciences. 2016;3:43-47.

17. Jahromi MAM, Al-Musawi S, Pirestani M, et al. Curcumin-loaded Chitosan Tripolyphosphate Nanoparticles as a safe, natural and effective antibiotic inhibits the infection of Staphylococcus aureus and Pseudomonas aeruginosa in vivo. Iran J Biotech. 2014;12(3):e1012.

18. Bhawana, Basniwal RK, Buttar HS, et al. Curcumin nanoparticles:preparation, characterization, and antimicrobial study. $J$ Agric Food Chem. 2011;59(5):205-2061.

19. Shlar I, Poverenov E, Vinokur Y, et al. High-throughput Screening of Nanoparticle-stabilizing Ligands: Application to Preparing Antimicrobial Curcumin Nanoparticles by Antisolvent Precipitation. Nano-Micro Letters. 2015;7(1):68-79.

20. Adahoun MA, Al-Akhras MAH, Jaafar MS, et al. Enhanced anti-cancer and antimicrobial activities of curcumin nanoparticles. Artif Cells Nanomed Biotechnol. 2017;45(1):98-107. 